Ann. Biol. anim. Bioch. Biophys., Ig63, 3 (3), 255-262

\title{
EFFICACITÉ DE QUELQUES PROTIDES ALIMENTAIRES CHEZ LE PORC
}

\author{
III. - ÉVOLUTTON DE L'EXCRÉTION AZOTÉE URINAIRE \\ DURANT LA PHASE DE RÉALIMENTATION, APRÈS UN JEÛNE PROTÉIQUE
}

J. DELORT-LAVAL, Geneviève CHARLET-LERY, K. DOGAN

avec la collaboration technique de Michèle Fiszlewicz et A. Roustan

Laboratoire de Recherches sur la Conservation et l'Efficacité des Aliments, 16, rue Claude-Bernard, Paris $5^{\mathrm{e}}$

\section{SOMMAIRE}

La détermination de la valeur biologique des protéines d'un aliment nécessite une estimation des dépenses endogènes en inanition azotée. Mais l'influence du jeûne protéique sur les bilans azotés ultérieurs est peu connue chez le Porc.

L'étude entreprise pour élucider ce problème porte sur 3 porcs d'environ $40 \mathrm{~kg}$, soumis avant et après 17 jours d'alimentation ternaire $(0,47$ à $0,54 \mathrm{~g}$ d'azote $/ \mathrm{kg}$ matière sèche), à un régime azoté apportant too $\mathrm{g}$ de $\mathrm{m}$. a. d. (farine de harengs) à l'u. f. et distribué en fonction du poids croissant et de l'appétit des animaux. Elle a permis de mettre en évidence que :

- le rapport $\mathrm{N}$ urinaire/ $\mathrm{N}$ ingéré ne rejoint que vers le $18^{\mathrm{e}}$ jour la courbe caractéristique de l'évolution de ce rapport en fonction du poids de l'animal;

- l'excrétion d'azote uréique et ammoniacal rapportée à l'azote ingéré augmente rapidement au début de la réalimentation azotée et ne se stabilise qu'au bout d'environ 20 jours.

La durée de la période de réalimentation azotée après le jeûne protéique ne doit donc pas être inférieure à 20 jours, pour qu'il soit possible d'établir un bilan azoté sur un animal en état nutritionnel normal.

La longueur de cette "période d'ajustement " rend souhaitable le rejet du jeûne protéique à la fin d'une expérience de mesure de valeur biologique chez le porc.

\section{INTRODUCTION}

La mesure de la valeur biologique d'une protéine par la méthode de THomasMitchel, (MITCHeil, I923-I924) exige l'établissement de bilans complets d'azote sur des animaux en état de réplétion et d'inanition protéique. Mais la position 
relative de ces périodes de mesure successives dans un schéma expérimental n'est pas indifférente.

Dans l'inanition protéique, l'évolution de l'excrétion d'azote peut en fait être répartie en 2 phases bien distinctes :

- l'une, de décroissance rapide correspondant à la transition entre le régime azoté normal et le régime protéiprive ;

- l'autre, de diminution à peine sensible et de longue durée, que Mourot (I935) appelle " période d'état ".

La phase de décroissance rapide varie selon les espèces : Io à I 2 jours chez le mouton recevant un régime pauvre en azote (MILLER et MoRRIson, I942), 3 jours chez le rat (Chick et al., I935), 7 jours chez le porc (ZeitTer et Charlet-L,erry, I96r), et en fonction du régime (Colombus, 1950). Le fait de ne pas atteindre un plateau constant d'excrétion endogène avant 20 jours (SMUTS, I935) n'empêche pas d'obtenir des valeurs comparables d'une expérience à l'autre, même après une inanition protéique de moins longue durée (Mrtchel, et al., I936). Il est donc possible chez le porc d'effectuer une mesure correcte des dépenses endogènes à partir du $8^{\mathrm{e}}$ jour de régime sans azote.

La phase de réalimentation azotée qui succède à ce jeûne se caractérise par une reprise rapide du poids et de la croissance des structures protéiques (MoligaARD, I955) et par un taux très élevé d'utilisation des protéines du régime ; mais on ignore la durée de cet anabolisme intense.

$\mathrm{Au}$ cours d'une expérience de réalimentation azotée après un jeûne protéique chez le mouton, MILLER et MORRISON (I942) ont établi une série de bilans par périodes successives de 9 jours; ils constatent entre les rétentions azotées des périodes I, II et III, des différences significatives; par contre, les résultats sont pratiquement similaires pour les périodes III et IV. Il est ainsi possible de mettre en évidence, chez le mouton, la nécessité d'une période de réalimentation azotée d'environ 20 jours après un jeûne protéique, avant de pouvoir effectuer une mesure de rétention azotée dans des conditions normales.

Nous avons entrepris des études analogues en vue de préciser les conséquences de la réalimentation azotée après un jeûne protéique chez le porc.

\section{MATÉRIEL, ET MÉTHODES}

A) Animaux

Les observations sont effectuées sur 3 porcs mâles castrés, de race Large White provenant d'une même portée. Ils pèsent respectivement $4 \mathrm{I}, 9-4 \mathrm{I}, 0-40,4 \mathrm{~kg}$ à l'âge de 128 jours après $\mathrm{I} 7$ jours de jeûne protéique et sont placés en cages à métabolisme individuelles.

\section{B) Régime alimentaire}

Le jeûne protéique est obtenu par la consommation d'un aliment ternaire dont la teneur en azote est comprise entre $0,47 \mathrm{~g}$ et $0,54 \mathrm{~g}$ par $\mathrm{kg}$ de matière sèche et dont la composi- 
tion ( ${ }^{1}$ ) est légèrement différente de la formule indiquée dans un mémoire antérieur (ZELTER et CHARLET-LERY, Ig6i).

En réplétion azotée, cet aliment est complété par de la farine de hareng de Norvège, de façon à atteindre un taux moyen de $20,6 \mathrm{~g} \mathrm{~N}$ par $\mathrm{kg}$ de matière sèche, soit environ Ioo g de matières azotées digestibles par unité fourragère.

Les repas sont distribués deux fois par jour, à l'exception de ceux du dimanche soir, les $2^{\mathrm{e}}$, $9^{\mathbf{e}}$ et $16^{\mathrm{e}}$ jour de la période de réalimentation azotée, qui sont respectivement le $2^{\mathbf{e}}$ jour de la périodé A I et les premiers jours des périodes A III et A V (tabl. 1). Les refus, le plus souvent insignifiants, sont pesés après chaque repas. Le niveau d'ingestion de matière sèche est réajusté les $2^{\mathbf{e}}$, ro ${ }^{\mathbf{e}}$ et I $8^{\mathrm{e}}$ jour de la phase de réalimentation azotée, en fonction du poids et de l'appétit des animaux, ce qui entraîne une répartition de cette phase en périodes de durée variable (tabl. $r$ et 3 ).

\section{C) Schéma expérimental}

L'expérience s'est déroulée selon le schéma décrit dans le tableau I.

TABLEAU I

Schéma expérimental

\begin{tabular}{|c|c|c|c|c|}
\hline \multirow{2}{*}{ Période } & \multirow{2}{*}{ Phase nutritionnelle } & \multirow{2}{*}{$\begin{array}{l}\text { M. A. T. }\left({ }^{1}\right) \\
\% \text { M. S. }\end{array}$} & \multicolumn{2}{|c|}{ Durée des périodes } \\
\hline & & & $\begin{array}{l}\text { Adaptation } \\
\text { (minimum) (j) }\end{array}$ & Bilans (j) \\
\hline 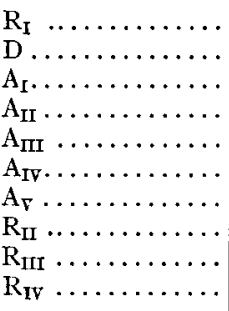 & $\begin{array}{l}\text { Réplétion azotée } \\
\text { Déplétion azotée } \\
\text { Réalimentation azotée } \\
\text { Réalimentation azotée } \\
\text { Réalimentation azotée } \\
\text { Réalimentation azotée } \\
\text { Réalimentation azotée } \\
\text { Réplétion azotée } \\
\text { Réplétion azotée } \\
\text { Réplétion azotée }\end{array}$ & $\begin{array}{c}12,8 \% \\
\neq 0 \\
12,8 \%\end{array}$ & $\begin{array}{c}(20) \\
8 \\
8\end{array}$ & $\begin{array}{l}8 \\
8 \\
5 \\
3 \\
4 \\
3 \\
5 \\
8 \\
8 \\
8\end{array}$ \\
\hline
\end{tabular}

M.A.T. = Matières azotées totales

\section{D) Techniques de collecte et analyses}

Les appareils utilisés au laboratoire pour la collecte séparée des excreta (ZELTER et CharletLERY, I961) sont mal tolérés par l'animal pendant des périodes trop prolongées. Mais les cages où sont placés les animaux permettent, durant les 20 jours de la phase de réalimentation azotée ( $A_{I}$ à $A_{\nabla}$ ), une récolte très correcte de l'urine, dans des récipients contenant de l'acide sulfurique, pour éviter tout dégagement d'ammoniac. Les fèces ne sont pas recueillis durant cette phase.

(1) Par $\mathrm{kg}:$ amijel : $500 \mathrm{~g}$; cérélose : $250 \mathrm{~g}$; poudre de papier-filtre : $100 \mathrm{~g}$; sucre de betterave: $40 \mathrm{~g}$ huile de maîs : $30 \mathrm{~g}$; mélange minéral : $80 \mathrm{~g}$. (Composition centésimale : $\mathrm{CaPO}_{4} \mathrm{H},{ }_{2} \mathrm{H}_{2} \mathrm{O}: 55 ; \mathrm{CaCO}_{3}: 20$; $\mathrm{NaCl}: 7,5 ; \mathrm{MgCO}_{3}: 7,5 ; \mathrm{Mg} \mathrm{SO}{ }_{4}: 6,0 ; \mathrm{K}_{2} \mathrm{CO}_{3}: \mathrm{I}, 8 ; \mathrm{Fe} \mathrm{SO}_{4}, 7 \mathrm{H}_{2} \mathrm{O}: 1,5 ; \mathrm{KI}: 0,47 ; \mathrm{CuSO}_{4}, 5 \mathrm{H}_{2} \mathrm{O}: 0, \mathrm{I} 7$; $\left.\mathrm{CO}\left(\mathrm{NO}_{3}\right)_{2}, 6 \mathrm{H}_{2} \mathrm{O}: 0,03 ; \mathrm{MnSO}_{4}: 0,03.\right)$

Vitamines : par jour, 5400 UI vit. A et 900 UI vit. $\mathrm{D}_{2}$; par kg de matière sèche ingérée : thiamine : $3,3 \mathrm{mg}$; riboflavine : $3,3 \mathrm{mg}$; ac. nicotinique : I I, $0 \mathrm{mg}$; pantothénate de $\mathrm{Ca}: \mathrm{I} 7,7 \mathrm{mg}$; pyridoxine : $5,5 \mathrm{mg}$; inositol : $9 \mathrm{mg}$; biotine : 0,15 mg; ac. folique : I5 $\mathrm{mg}$; acide p. aminobenzoïque : $20 \mathrm{mg}$; chlorhydrate de choline : $500 \mathrm{mg}$; acide ascorbique : $73 \mathrm{mg}$.

Annales de Biologie animale. -- I963. 
Par contre, le port de ces appareils rend possible la collecte séparée des fèces et de l'urine lors de l'établissement des bilans complets $\left(\mathrm{R}_{\mathrm{I}}, \mathrm{D}, \mathrm{R}_{\mathrm{II}}, \mathrm{R}_{\mathrm{II}}, \mathrm{R}_{\mathrm{IV}}\right)$.

Les analyses sont effectuées tous les quatre jours en périodes de bilans et chaque jour durant la phase de réalimentation. Les dosages portent sur l'azote total des aliments, de l'urine et des fèces frais par la méthode de KJELDAHL (1), sur l'azote ammoniacal de l'urine par microdiffusion à froid (CONWAY, I950) et sur l'urée urinaire par la méthode colorimétrique de SCHRAMm et AiNES (1959).

TABLEAU 2

Résultats moyens journaliers par période et par animal

\begin{tabular}{|c|c|c|c|c|c|c|c|c|c|c|}
\hline Période & $\begin{array}{c}\text { Durée } \\
\text { (jours) }\end{array}$ & Animal & $\begin{array}{c}\text { Poids } \\
(\mathrm{kg})\end{array}$ & $\begin{array}{c}\text { M.S. i ( }{ }^{(1)} \\
(\mathrm{g})\end{array}$ & $\begin{array}{c}\text { M.S.i. }\left({ }^{(1)}\right) \\
/ 1^{00} .7\end{array}$ & $\begin{array}{c}\text { N ingéré } \\
\text { (g) }\end{array}$ & $\begin{array}{l}\text { N fécal } \\
\text { (g) }\end{array}$ & $\begin{array}{c}\mathrm{N} \text { fécal } \\
\% \\
\text { M.S.f. (1) }\end{array}$ & $\begin{array}{c}N \text { uri- } \\
\text { naire } \\
\text { total }(g)\end{array}$ & $\begin{array}{c}\mathrm{N} \text { bilan } \\
\text { applarent } \\
\text { (g) }\end{array}$ \\
\hline $\mathrm{R}_{\mathrm{I}}$ & 8 & $\begin{array}{l}E \\
F \\
G\end{array}$ & $\begin{array}{l}38,0 \\
37,3 \\
36,4\end{array}$ & $\begin{array}{l}1370 \\
1290 \\
1251\end{array}$ & $\begin{array}{l}105,0 \\
102,5 \\
100,2\end{array}$ & $\begin{array}{l}24,92 \\
24,33 \\
23,51\end{array}$ & $\begin{array}{l}3,01 \\
3,38 \\
3,25\end{array}$ & $\begin{array}{l}1,47 \\
1,84 \\
1,67\end{array}$ & $\begin{array}{l}7,48 \\
7,49 \\
7,27\end{array}$ & $\begin{array}{l}14,43 \\
13,46 \\
12,99\end{array}$ \\
\hline D & 8 & $\begin{array}{l}\mathrm{E} \\
\mathrm{F} \\
\mathrm{G}\end{array}$ & $\begin{array}{l}11,4 \\
40,0 \\
39,7\end{array}$ & $\begin{array}{l}1480 \\
1415 \\
1434\end{array}$ & $\begin{array}{l}109,2 \\
107,0 \\
109,0\end{array}$ & $\begin{array}{l}0,77 \\
0,74 \\
0,75\end{array}$ & $\begin{array}{l}2,60 \\
2,52 \\
2,75\end{array}$ & $\begin{array}{l}1,11 \\
1,08 \\
1,17\end{array}$ & $\begin{array}{l}2,50 \\
2,28 \\
2,18\end{array}$ & $\begin{array}{l}-4,33 \\
-4,06 \\
-4,18\end{array}$ \\
\hline$A_{I}$ & 5 & $\begin{array}{l}\mathrm{E} \\
\mathrm{F} \\
\mathrm{G}\end{array}$ & $\begin{array}{l}41,8 \\
42,5 \\
42,4\end{array}$ & $\begin{array}{l}1526 \\
1517 \\
1497\end{array}$ & $\begin{array}{l}10^{\prime}, 2 \\
109,9 \\
108,6\end{array}$ & $\begin{array}{l}25,84 \\
26,05 \\
25,26\end{array}$ & & & $\begin{array}{l}5,63 \\
5,43 \\
5,76\end{array}$ & \\
\hline$A_{I I}$ & 3 & $\begin{array}{l}\mathrm{E} \\
\mathrm{F} \\
\mathrm{G}\end{array}$ & $\begin{array}{l}47,9 \\
45,8 \\
45,2\end{array}$ & $\begin{array}{l}1744 \\
1640 \\
1627\end{array}$ & $\begin{array}{l}116,2 \\
112,8 \\
112,9\end{array}$ & $\begin{array}{l}36,61 \\
37,10 \\
3 \nmid, 09\end{array}$ & & & $\begin{array}{r}8,74 \\
8,98 \\
11,28\end{array}$ & \\
\hline$A_{\text {III }}$ & 4 & $\begin{array}{l}\text { E } \\
F \\
G\end{array}$ & $\begin{array}{l}50,3 \\
48,2 \\
47,3\end{array}$ & $\begin{array}{l}1609 \\
1620 \\
1586\end{array}$ & $\begin{array}{l}103,6 \\
107,5 \\
106,7\end{array}$ & $\begin{array}{l}34,32 \\
34,19 \\
33,12\end{array}$ & & & $\begin{array}{l}7,49 \\
9,6^{\prime} \\
7,90\end{array}$ & \\
\hline$A_{I V}$ & 3 & $\begin{array}{l}\text { E } \\
\text { F } \\
\text { G }\end{array}$ & $\begin{array}{l}53,5 \\
51,4 \\
50,1\end{array}$ & $\begin{array}{l}1894 \\
1878 \\
1851\end{array}$ & $\begin{array}{l}116,8 \\
119,2 \\
119,6\end{array}$ & $\begin{array}{l}40,09 \\
39,51 \\
38,59\end{array}$ & & & $\begin{array}{r}9,68 \\
10,93 \\
13,05\end{array}$ & \\
\hline$A_{V}$ & 5 & $\begin{array}{l}\text { E } \\
\text { F } \\
G\end{array}$ & $\begin{array}{l}56,6 \\
54,6 \\
52,9\end{array}$ & $\begin{array}{l}1834 \\
1781 \\
1751\end{array}$ & $\begin{array}{l}108,8 \\
108,3 \\
108,8\end{array}$ & $\begin{array}{l}39,21 \\
37,49 \\
36,9 !\end{array}$ & & & $\begin{array}{l}12,23 \\
12,26 \\
10,92\end{array}$ & \\
\hline $\mathrm{R}_{\text {II }}$ & 8 & $\begin{array}{l}\text { E } \\
F \\
G\end{array}$ & $\begin{array}{l}61,9 \\
59,7 \\
57,0\end{array}$ & $\begin{array}{l}2092 \\
1.989 \\
1940\end{array}$ & $\begin{array}{l}116,5 \\
113,5 \\
111,5\end{array}$ & $\begin{array}{l}45,99 \\
43,66 \\
42,47\end{array}$ & $\begin{array}{r}5,97 \\
5,24 \\
4,87\end{array}$ & $\begin{array}{l}1,64 \\
1,59 \\
1,57\end{array}$ & $\begin{array}{l}17,20 \\
15,58 \\
16,06\end{array}$ & $\begin{array}{l}22,82 \\
22,84 \\
21,54\end{array}$ \\
\hline $\mathrm{R}_{\text {III }}$ & 8 & $\begin{array}{l}\text { E } \\
\text { F } \\
\text { G }\end{array}$ & $\begin{array}{l}76,3 \\
7 / \mathbf{k}, \mathbf{4} \\
70,8\end{array}$ & $\begin{array}{l}2439 \\
2345 \\
2187\end{array}$ & $\begin{array}{l}117,3 \\
11 / 4,8 \\
110,9\end{array}$ & $\begin{array}{l}50,92 \\
48,15 \\
45,02\end{array}$ & $\begin{array}{l}7,59 \\
6,0^{\prime} \\
5,72\end{array}$ & $\begin{array}{l}1,8 \\
1,73 \\
1,6^{\prime} \mathrm{k}\end{array}$ & $\begin{array}{l}25,68 \\
21,16 \\
18,72\end{array}$ & $\begin{array}{l}17,65 \\
20,65 \\
20,58\end{array}$ \\
\hline $\mathrm{R}_{\mathrm{VI}}$ & 8 & $\begin{array}{l}\mathrm{E} \\
\mathrm{F} \\
\mathrm{G}\end{array}$ & $\begin{array}{l}88,7 \\
85,7 \\
80,7\end{array}$ & $\begin{array}{l}2574 \\
2434 \\
2379\end{array}$ & $\begin{array}{l}111,1 \\
117,9 \\
110,0\end{array}$ & $\begin{array}{l}52,03 \\
50,2 ' t \\
50,12\end{array}$ & $\begin{array}{l}6,98 \\
6,57 \\
6,77\end{array}$ & $\begin{array}{l}1,85 \\
1,82 \\
1,58\end{array}$ & $\begin{array}{l}30,40 \\
26,51 \\
26,81\end{array}$ & $\begin{array}{l}11,65 \\
17,16 \\
16,54\end{array}$ \\
\hline
\end{tabular}

(1) M. S. i. = Matière sèche ingérée. M. S. f. = Matière sèche fécale.

(1) Catalyseur : Oxyde rouge de mercure. Microdistillation dans l'appareil de Parnas-Wagner. Le distillat recueilli dans une solution d'acide borique à I p. Ioo est titré par $\mathrm{SO}_{4} \mathrm{H}_{2} \mathrm{~N} / 50$, en présence de l'indicateur de Conway (I950). 


\section{RÉSULTATS}

Les résultats moyens périodiques sont portés dans le tableau 2. Les animaux, ont, durant toute 1'expérience, un état de réplétion en matière sèche $\frac{(\text { M. S. i) }}{\mathrm{P}^{0,70}}$ relativement constant et toujours suffisant pour assurer une croissance régulière.

L'excrétion urinaire d'azote qui subit, lors de la suppression des protéines alimentaires, une chute brutale, augmente très rapidement et avec régularité dès le début de la réalimentation azotée.

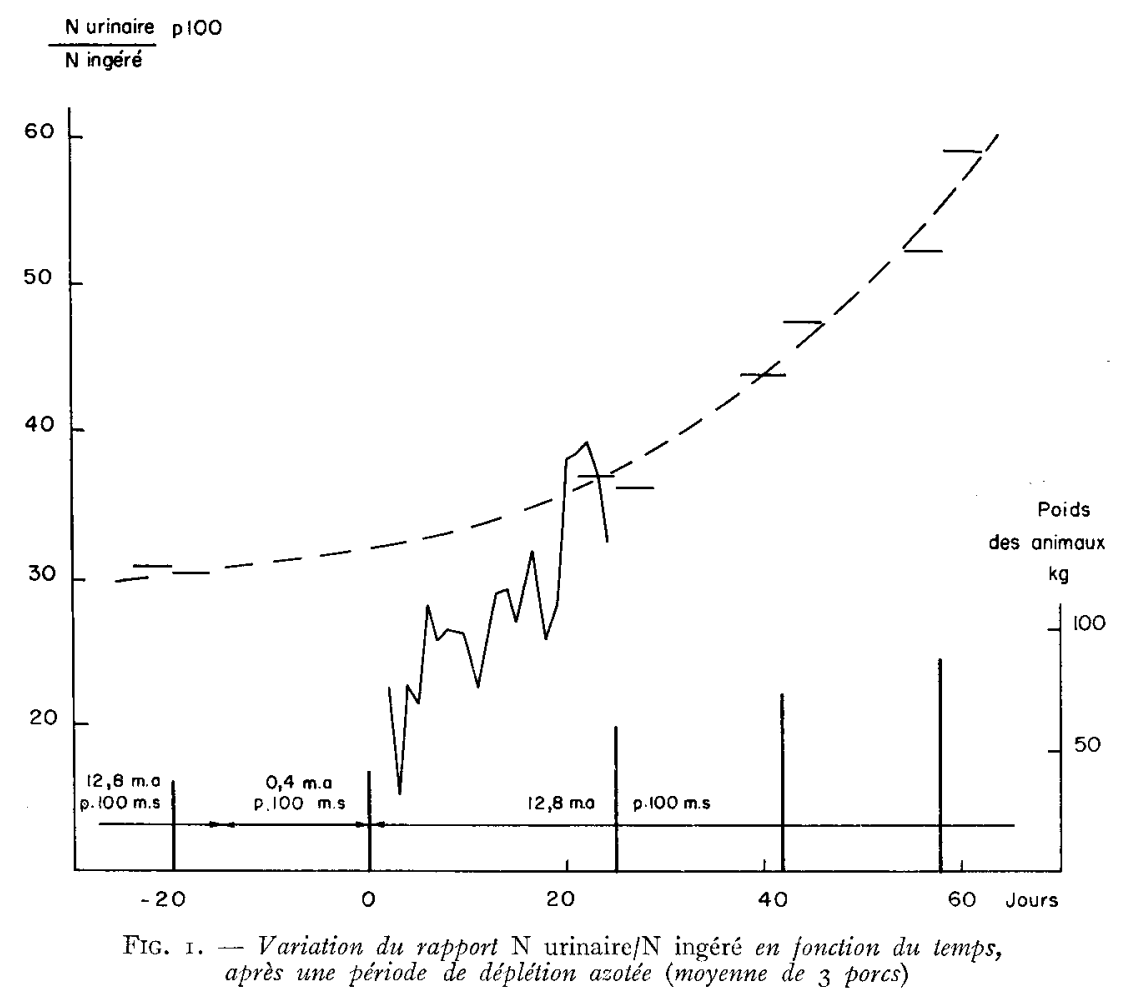

Comme l'ingestion des protides s'accroît régulièrement, l'excrétion d'azote brut n'a pas de signification physiologique; par contre, le rapport $\mathrm{N}$ urinaire/ $\mathrm{N}$ ingéré permet de suivre, en fonction du temps, l'évolution de l'anabolisme protidique de I'animal (fig. I).

L'examen de cette courbe montre qu'il faut environ 20 jours pour que l'excrétion urinaire d'azote de la période de réalimentation rejoigne le niveau atteint durant les phases de réplétion qui l'encadrent.

Cependant, la quantité totale d'azote de l'urine ne mesure que l'importance globale des pertes azotées de l'organisme; la répartition entre urée et ammoniaque 
(tabl. 3) fournit, sur l'orientation du métabolisme azoté, des indications plus précises.

\section{TABLEAU 3}

Évolution de la répartition de l'azote urinaire durant les phases successives de la période de réalimentation azotée (moyenne de 3 porcs)

\begin{tabular}{|c|c|c|c|c|c|c|c|}
\hline \multirow[b]{2}{*}{ Période (jours). . . . . } & \multirow{2}{*}{ Déplétion } & \multicolumn{6}{|c|}{ Réalimentation azotée } \\
\hline & & $1-7$ & $8-1 / t$ & $15-21$ & $22-28$ & $37-44$ & $53-60$ \\
\hline Ningéré $(g) \ldots \ldots \ldots$ & 0,76 & 28,76 & 35,63 & 38,98 & 41,20 & 48,03 & 50,51 \\
\hline Nurinaire $(g) \ldots \ldots$ & 2,32 & 6,81 & 9,50 & 12,40 & $16,0_{4}^{\prime}$ & 21,95 & 28,16 \\
\hline N bilan ........... & & 22 & 26 & 26 & 28 & 26 & 22 \\
\hline Nurée $(g) \ldots \ldots \ldots$ & 0,27 & 3,31 & 5,32 & 7,78 & 10,86 & 15,89 & 21,34 \\
\hline $\mathrm{N}-\mathrm{NH}_{3}(\mathrm{~g}) \ldots \ldots \ldots$ & 0,97 & 1,57 & 2,08 & 2,40 & 3,00 & 3,15 & 3,31 \\
\hline Nurée $\%$ N urinaire. & 11,5 & 48,6 & 56,0 & 62,7 & 67,7 & 72,4 & 75,7 \\
\hline $\begin{array}{l}\mathrm{N}-\mathrm{NH}_{3} \% \mathrm{~N} \text { urinaire. } \\
\mathrm{N} \text { urée }+\mathrm{N}-\mathrm{NH}_{3}\end{array}$ & 41,8 & 23,1 & 21,9 & 19,4 & 18,7 & 14,3 & 11,8 \\
\hline$\% \mathrm{~N}$ urinaire........ & 53,3 & 71,7 & 77,9 & 82,1 & 86,4 & 86,7 & 87,5 \\
\hline
\end{tabular}

Durant la phase de recouvrement du catabolisme normal, l'urée augmente très rapidement et parallèlement à l'excrétion d'azote total; l'accroissement de l'ammoniaque est beaucoup plus lent. La somme des azotes uréique et ammoniacal rapportée à l'azote urinaire total augmente régulièrement et tend à se stabiliser au-delà du vingtième jour. Cela confirme la constatation qui découle de l'examen du rapport entre azote ingéré et urinaire.

\section{DISCUSSION}

L'évolution de l'excrétion d'azote total de l'urine ou de certains de ses composants (urée et ammoniaque) met en évidence la nécessité d'une période de réalimentation azotée d'environ 20 jours, après un jeûne protéique de durée équivalente, pour rétablir le métabolisme normal de l'azote chez le Porc.

Mais si l'inanition protéique se prolonge beaucoup, de graves perturbations non réversibles sont à craindre, comme l'ont démontré Roche et al. (I938).

En période de réplétion azotée $\left(\mathrm{R}_{1}, \mathrm{R}_{\mathrm{II}}, \mathrm{R}_{\mathrm{II}}, \mathrm{R}_{\mathrm{IV}}\right)$ il est possible d'établir les corrélations entre les valeurs d'excrétion urinaire totale (NU.) ou de $\mathrm{N}$ urinaire/N ingéré, d'une part, et le poids (P) ou l'âge de l'animal, d'autre part. Les plus élevées de ces corrélations relient entre elles NU. et $\mathrm{P}\left(r=0,955 ; \mathrm{NU} .=0,44-\mathrm{I0,04}_{\mathrm{P}}\right)$ ou les $\log$ de ces 2 valeurs $(r=0,993 ; \log \mathrm{NU} .=\mathrm{I}, 63 \log \mathrm{P}-\mathrm{r}, 60)$. L'existence de ces corrélations s'expliquerait par le fait que l'azote urinaire dépend, pour sa part endogène, de la taille métabolique $\left(\mathrm{P}^{0,70}\right)$, et, pour sa part exogène, de la quantité de protéines ingérées, elle-même liée par l'intermédiaire de la matière sèche au poids vif de l'animal. C'est encore dans ce cas vers le I8-20 jour que les courbes expérimentales d'excrétion d'azote, dont l'allure est comparable à celle de la figure $\mathrm{I}$, rejoignent les droites de régression calculées ci-dessus. 
Nos observations sur le porc confirment donc les études de MILIER et MorRISON (I942) chez le mouton.

Pat contre, Armstrong et Mitchel (I955), par une analyse quotidienne de l'azote urinaire, fixent le début de la période de mesure de la valeur biologique au I3 $3^{\mathbf{e}}$ jours après la fin de l'inanition protéique, lorsque les porcs sont maintenus à un niveau de matière sèche constant durant toute la période. Cette méthodologie, et plus encore celle de NEHRING et LAUBE (I96I), ne sont pas exemptes de critiques : le maintien d'une ingestion de matière sèche constante durant de longues périodes a pour conséquence une diminution progressive de la couverture des besoins d'énergie de l'animal en croissance et risquerait d'entrainer une utilisation des protéines à des fins énergétiques et un amoindrissement de l'efficacité de l'azote ingéré (MIL LER et PAYNe, I96I).

Cette hypothèse est en accord avec les résultats de NJAA (I959) qui constate, chez le rat, que la valeur biologique d'une farine de hareng dans un régime de Io $\mathrm{p}$. Ioo de protéines est liée positivement aux taux d'ingestion de matière sèche de ce régime.

Il nous semble donc indispensable de maintenir un état constant de réplétion de matière sèche (M. S. i./ $\mathrm{P}^{0,70}$ ) à travers les différentes périodes expérimentales, ce qui rapproche les conditions de la mesure de celle d'une alimentation normale. Cette nécessité a pour conséquence l'allongement de la période de réalimentation azotée. Comme, en outre, la longueur de cette " phase d'ajustement " peut varier en fonction de la qualité des protéines ingérées (KoN, I928), il est souhaitable de rejeter le jê̂ne azoté à la fin des essais.

Toutefois, si l'expérience est trop longue, l'extrapolation des données mesurées en régime protéiprive peut devenir hasardeuse. De plus, à partir d'un certain taux de protéines dans la ration, la valeur biologique varie nettement en fonction de l'âge (BEIN, I96I ; HENRY and KON, I957).

Pour toutes ces raisons, la mise au point de schémas d'expérience courts, comprenant 2 (ou 3) périodes de bilans en réplétion azotée suivies d'une mesure des dépenses endogènes en régime protéiprive, présenterait des avantages indiscutables pour la précision des mesures.

Reçu pour publication en mai 1963.

\begin{abstract}
SUMMARY
THE EFFICIENCY OF SOME ALIMENTARY PROTIDS IN THE PIG.

III - THE DEVELOPMENT OF NITROGENOUS EXCRETION IN URINE DURING THE REFERDING PHASE AFTER PROTEIN STARVATION
\end{abstract}

Determination of the biological value of dietary proteins requires an estimation of the endogenous nitrogen losses during nitrogen starvation. However the influence of protein starvation on the subsequent nitrogen balance in the pig is little known.

The present study is set out to elucidate this probleme in three pigs each weighing about $40 \mathrm{~kg}$, subjected both before and after $\mathrm{I} 7$ days of $\mathrm{N}$-free diet ( 0.47 to $0.54 \mathrm{~g}$ nitrogen per $\mathrm{kg}$ of dry matter) to a regime with roo $\mathrm{g}$ d. c. p. (herring meal) per feed unit which was given according to the increasing weight and appetite of the pigs. The following conclusions can be drawn :

- The urinary $\mathrm{N}$ : ingested $\mathrm{N}$ ratio approaches the curve characteristic of the change of this ratio with the animal's weight, only towards the eighteenth day (fig. I). 
- The excretion of urea and ammonia nitrogen increases rapidly after the cessation of nitrogen starvation and only becomes stable after some zo days (table 3 ).

The lenght of nitrogenous refeeding after protein starvation must not therefore be less than zo days in order to determine the nitrogen balance of an animal in normal nutritional status.

The lenght of this period of adjustement makes desirable to put the period of protein starvation at the end of an experiment measuring a biological value in the pig.

\section{RÉFÉRENCES BIBLIOGRAPHIQUES}

Armstrong D. G., Mrtchell II. H., 1955. Protein nutrition and the utilisation of dietary protein at different levels of intake by growing swine. J. anim. Sci, 14, 49-68.

BEIN L., I961. N-Bilanz und Gesamtsoffwechselversuche an wachsenden Albinoratten. Arch. Tierernähr., 11, $42-70$.

Chick H., Hutcirson J. C. D., JACkson H. M., 1935. The biological value of proteins. VI Further investigations on the balance sheet method. VII The influence of variation in the level of protein in the diet and of heating the protein on its biological value. Biochem. J., 29, I 702-I 7 I I ; I I 2 - I 7 I9.

Colombus A., I950. Die Variabilität der biologischen Eiweisswertigkeit unter verschiedenen Bedingungen nach Stoffwechsel versuchen an wachsenden Schweinen. Arch. Tierernähr., 1, 38-55, 84-128.

Conway E. J., 1950. Microdiffusion analysis and volumetric error. 95-97, $3^{\ominus}$ éd., Crosby Lockwood and Son Ltd, London.

Henry Kathleen M., Kon S. K., i957. Iiffect of level of protein and of age of rat on the biological value of protein. Brit. J. Nutr., 11, 305-3I3.

KoN S. K., 1928. The nutritional value of tuberin, the globulin of potato. Biochem. J., 22, $26 \mathrm{I}-267$.

Miller D. S., PAYNe P. R., I96I. The effect of calorie intake on net dietary protein value. Proc. Nutr. Soc., 20, XLVIII-XL IX.

Miller J. I., Morrison F. B., 1942. The influence of feeding low nitrogen rations on the reliability of biological values. J. Agric. Res., 65, 429-45I.

Mitciele II. H., I923-24. A method of determining the biological value of protein. J. Biol. Chem., 58, 873 et 903 .

Mitchelt H. H., Burrows W., Beadles J. R., I936. The significance and accuracy of biological values of proteins computed from nitrogen metabolism data. J. Nutr., 11, 257-274.

Mollgard II., I955. Les méthodes de détermination quantitative de la croissance. Essais des facteurs externes et internes. 282 .beretning fra forsogslaboratoriet. I-I go. Statens Flusdyrbrugsudvalg, Copenhague. (Danois)

Mourot G., 1935. La répartition des représentants urinaires du métabolisme azoté et sa signification physiologique. VII. L'évolution du métabolisme azoté endogène spécifique au cours du jeûne protéique. Bull. Soc. Chim. Biol. Paris, 17, I 741-1789.

NEnRING K., LAUbE W., r961. Untersuchungen über die biologische Wertigkeit des Eiweisses verschiedener Futterstoffe beim wachsenden Schwein. Z. Tierernähr. Tierphysiol., 16, I21-г 32.

NJAA L. R., r959. Biological value of herring meal protein. Urinary nitrogen excretion in relation to protein content of diet and food intake. Brit. J. Nutr., 13, I 42-I 5 O.

Rocile Andrée, Rocie J., Drouineau Simone, Passelaigue P., i938. Sur la réparation de pertes azotées importantes et couverture des besoins alimentaires en protides. I. Bull. Soc. Chim. Biol. Paris, $20,217-25 \mathrm{I}$.

Schramm G., Aines P. D., 1959. Colorimetric determination of urease activity in soybean meal. J.amer. Oil Chem. Soc., 36, I-3.

Syuts D. B., 1935. The relation between the basal metabolism and the endogenous nitrogen metabolism with particular reference to the estimation of the maintenance requirement of protein. $J . N u i r ., 9,4^{\circ} 3^{-}$ 433 .

Zelter S. Z., Charlet-Lery Geneviève, r96i. Efficacité de quelques protides alimentaires chez le Porc I Excrétion d'azote métabolique fécal et endogène urinaire. Ann. Biol. anim. Bioch. Biophys., 1,. $29-46$ 